\title{
Response to Letter to the Editor: Improving information to caregivers of cancer patients: the Herlev Hospital Empowerment of Relatives through More and Earlier information Supply (HERMES) randomized controlled trial
}

\author{
Line Lund ${ }^{1}$ (D) $\cdot$ Lone Ross $^{1}$ (D) $\cdot$ Morten Aagaard Petersen ${ }^{1}$ (D) $\cdot$ Lisa Sengelov ${ }^{2} \cdot$ Mogens Groenvold $^{1,3}$ (D)
}

Received: 26 September 2019 / Accepted: 4 October 2019 / Published online: 13 November 2019

(C) Springer-Verlag GmbH Germany, part of Springer Nature 2019

\section{Dear Editor,}

We thank Kajiwara et al. for their interest in our work [1] and for their comments [2].

We agree with Kajiwara et al. that it is of great importance to focus on simple interventions that can easily be provided to the caregivers; this is in the interest of both the busy health care professionals and the often very burdened caregivers who may not have the energy to engage in complex interventions.

In our experience, face-to-face interventions are preferable, but we also agree that delivering an intervention via telephone is worth considering as a supplement if this is the most practical way to ensure that the caregivers receive given the intervention.

As Kajiwara et al. highlight, we conducted a short-term study [1], and we agree that it is possible that there could be effects of the intervention on anxiety and depression that would only be revealed by a long-term follow-up. This could be interesting to investigate in future studies.

Line Lund

line.lund.01@regionh.dk

1 The Research Unit, Department of Palliative Medicine, Bispebjerg and Frederiksberg Hospital and University of Copenhagen, Bispebjerg Bakke 23, DK-2400 Copenhagen, NV, Denmark

2 The Department of Oncology, Herlev and Gentofte Hospital and University of Copenhagen, Herlev Ringvej 75,

DK-2730 Herlev, Denmark

3 Section of Health Services Research, Department of Public Health, University of Copenhagen, Øster Farimagsgade 5,

DK-1014 Copenhagen K, Denmark
Even though we have developed and tested our intervention among caregivers of cancer patients only, the intervention may also have positive effects in other groups of caregivers, e.g., among caregivers of people with dementia. An investigation of this should start out by evaluating the intervention in qualitative studies in these groups to ensure that the content of the intervention is relevant. It is possible that some of the 14 questions are not relevant or that important questions are lacking.

\section{Compliance with ethical standards}

Conflict of interest All authors declare that they have no conflict of interest.

\section{References}

1. Lund L, Ross L, Petersen M, Sengelov L, Groenvold M (2019) Improving information to caregivers of cancer patients: the Herlev Hospital Empowerment of Relatives through More and Earlier information Supply (HERMES) randomized controlled trial. Support Care Cancer:1-12. https://doi.org/10.1007/s00520-019-04900-3

2. Kajiwara K, Kako J, Noto H, Oosono Y, Kobayashi M (2019) Letter to the editor: "Improving information to caregivers of cancer patients: the Herlev Hospital Empowerment of Relatives through More and Earlier information Supply (HERMES) randomized controlled trial". Support Care Cancer:1-2. https://doi.org/10.1007/ s00520-019-04995-8

Publisher's note Springer Nature remains neutral with regard to jurisdictional claims in published maps and institutional affiliations. 